\title{
Systematic analysis of the radiologic findings of aortic dissections on unenhanced postmortem computed tomography
}

\author{
Garyfalia Ampanozi - Patricia M. Flach • Juergen Fornaro • \\ Steffen G. Ross • Wolf Schweitzer • Michael J. Thali • \\ Thomas D. Ruder
}

Accepted: 6 January 2015/Published online: 28 February 2015

(C) Springer Science+Business Media New York 2015

\begin{abstract}
The aim of this study was to evaluate the diagnostic criteria and to identify the radiological signs (derived from known radiological signs) for the detection of aortic dissections using postmortem computed tomography (PMCT). Thirty-three aortic dissection cases were retrospectively evaluated; all underwent PMCT and autopsy. The images were initially evaluated independently by two readers and were subsequently evaluated in consensus. Known radiological signs, such as dislocated calcification and an intimomedial flap, were identified. The prevalence of the double sedimentation level in the true and false lumen of the dissected aorta was assessed and defined as a postmortem characteristic sign of aortic dissection. Dislocated calcification was detected in $85 \%$ of the cases with aortic calcification; whereas in $54 \%$ of the non-calcified aortas, the intimomedial flap could also be recognized. Double sedimentation was identified in 16/33 of the cases. Overall, in $76 \%$ (25/33) of the study cases, the described signs, which are indicative for aortic dissection, could be identified. In this study, three diagnostic criteria of aortic dissection were identified using non-enhanced PMCT images of autopsy-confirmed dissection cases.
\end{abstract}

G. Ampanozi $(\bowtie) \cdot$ P. M. Flach · J. Fornaro .

S. G. Ross - W. Schweitzer - M. J. Thali - T. D. Ruder

Department of Forensic Medicine and Imaging, Institute of Forensic Medicine, University of Zurich, Winterthurerstrasse 190/52, 8057 Zurich, Switzerland e-mail: garyfalia.ampanozi@virtopsy.com;

garyfalia.ampanozi@irm.uzh.ch; ampanozi@hotmail.com

T. D. Ruder

Institute of Diagnostic, Interventional and Pediatric Radiology, Inselspital, University Hospital Bern, Freiburgstrasse 4, 3010 Bern, Switzerland
Keywords Aortic dissection - Postmortem computed tomography · Postmortem imaging · Forensic radiology · Virtopsy

\section{Introduction}

Aortic dissection is considered to be an acute aortic syndrome [1] and is the most common acute emergency condition of the aorta [2]. The outcome of this condition is determined by the type and extent of the dissection and the subsequent complications, such as aortic rupture [2]. Determining the location and extent of an aortic dissection is essential for its classification and treatment. The most widely used system is the Stanford classification in type A (involving the ascending aorta) and type $\mathrm{B}$ (dissection limited to the aorta distal to the left subclavian artery) dissections [3]. The former presents a surgical emergency, whereas the latter, if uncomplicated, can be treated medically [4-7].

An intimal tear is considered to be causal for classical aortic dissections $[8,9]$. An aortic dissection is typically characterized by an intimomedial flap, which leads to the formation of a double (true and false) lumen in the aorta [8]. Further types of aortic dissections (triple lumen aortic dissection with the Mercedes-Benz sign or double barreled aorta with circumferential intimomedial flap [10]) are also being described. Degeneration of the medial layer of the aortic wall, inheritable disorders of the elastic layer and hypertension, are possible etiologies [9]. Furthermore, pregnancy [8], previous aortic surgery, age, aortic coarctation [11, 12], inflammatory diseases, cocain abuse, trauma, and a bicuspid aortic valve [11] are also considered risk factors. Atherosclerosis and calcification of the aorta typically affects both the intima and media layers of the aortic wall [13]. Subsequently, dissection of the aorta and 
elevation of the intimomedial layer will cause the radiologically described sign of displaced intimal calcification observed in clinical practice $[1,5,6,10,14-16]$.

Rupture of the aortic dissection may lead to hemothorax, hemopericardium, abdominal or retroperitoneal hemorrhage [14] and subsequent death due to fatal bleeding or pericardial tamponade. Another cause of death in type A aortic dissections is myocardial ischemia due to the involvement of the coronary artery origins in the dissection [17]. Sato et al. [18] described the rare incidence of a prolapsing intimal flap through the aortic valve with myocardial ischemia and aortic regurgitation as a possible fatal condition. Aortic insufficiency due to aortic dissection can also cause death [10].

Postmortem imaging, particularly postmortem computed tomography (PMCT), is used in forensic institutes across the world, either as a triage procedure, complementary to an autopsy or even, in some cases, as a substitution for autopsy $[19,20]$. PMCT has been proven to be excellent for detecting foreign bodies, osseous fractures, and gas collections [21], but it lacks specificity if soft tissue lesions, including vascular pathologies, are suspected [22]. Normal postmortem findings should be taken into account to correctly interpret PMCT images. These are well described in the literature, and many are relevant to the vascular system (hyperattenuating aortic wall [23], sedimentation of blood [24, 25]). The diagnostic signs of aortic dissections on non-contrasted PMCT images have never been examined systematically. They are either described sporadically as case reports or in articles involving PMCT angiography (PMCTA) [22, 26-28]. Currently, only certain forensic centers are able to perform PMCTA; therefore, unenhanced PMCT is most widely used and interpreted in postmortem radiology.

The aim of this study was to systematically describe the findings of aortic dissections using unenhanced PMCT.

\section{Materials and methods}

The database from the Institute of Zurich was evaluated for a study period of 51 months. During this time frame, 2123 autopsy cases were investigated. Thirty-three cases of nontraumatic aortic dissection, both with and without subsequent aortic rupture, were retrospectively identified. The study population included 19 males and 14 females. The mean age at the time of death was 61.4 years (standard deviation, 13.9 years; median, 64 years). All of the included cases underwent unenhanced PMCT examination and a subsequent autopsy, which was performed by two forensic pathologists, at least one of which was board certified. All of the postmortem examinations, including the imaging, were commissioned by the responsible local justice authorities.
Imaging parameters

The postmortem whole body imaging was carried out in the supine position. Imaging was performed on a dual-source CT scanner (SOMATOM ${ }^{\circledR}$ Definition Flash, Siemens Medical Solutions, Forchheim, Germany). Imaging parameters were set at the values recommended in the recent literature [29]: tube voltage was $120 \mathrm{kVp}$, slice collimation was $128 \times 0.6 \mathrm{~mm}$. All scans consisted of a whole body PMCT scan plus a dedicated head and neck scan, as well as a dedicated chest and abdomen scan. All of the scans (whole body, head and neck, chest, and abdomen) were performed using the automatic dose modulation software Siemens CARE Dose4D (Siemens Medical Solutions, Forchheim, Germany). All of the PMCT image reconstructions of the chest and abdomen were performed using the soft-tissue and lung window with soft and hard kernels, respectively.

\section{Assessment of the images}

A picture archiving and communication system (PACS, Sectra Workstation IDS7, Version 14.3.5.136, Sectra, Linkoping, Sweden) was used for radiological interpretation.

The Stanford classification of the type of dissection was applied and any signs of extravasation of blood (hemopericardium, hemothorax, hemoperitoneum) were also noted.

For each case, the presence of the following findings was assessed on PMCT: (1) aortic calcification; (2) dislocated intimal calcification, defined as an eccentric calcified flap that is displaced medially [30] (Fig. 1); (3) the presence of an intimomedial flap between the true and false lumen, when blood enters and separates the aortic medial layer [2] (Fig. 2); and (4) the presence of a double sedimentation sign, defined as either two distinct levels of sedimentation (within both the true and false lumen) or as single sedimentation line within either the true or the false lumen (i.e. the sedimentation is interrupted by the intimomedial flap) (Fig. 2).

Two readers (a radiologist and a forensic pathologist, each with 5 years of experience in postmortem imaging) evaluated the PMCT images. Findings (2) and (3) were initially read separately by each reader. They were then subsequently read in consensus. One reader (the forensic pathologist) repeated the image reading to assess the intra-reader reliability. Findings (1) and (4) were only read in consensus.

Statistical analysis

Dedicated statistical software was used (SPSS 20.0, IBM, Armonk, NY, USA) for analysis. The continuous variables were expressed as the means \pm standard deviations and the 
medians. The categorical variables are provided as frequencies and percentages. Kappa Cohen's test was used to assess the inter- and intra-reader agreement. The Chi square test was used to assess the statistical significance. A $p$ value of $<0.05$ was considered to be statistically significant.

Descriptive information regarding the study sample was retrieved from the scanning database.

\section{Results}

In this retrospective study, 33 aortic dissection cases were evaluated.

Using the general characteristics of the cases, 29 were classified as Stanford type A cases. In 18/33 cases, a

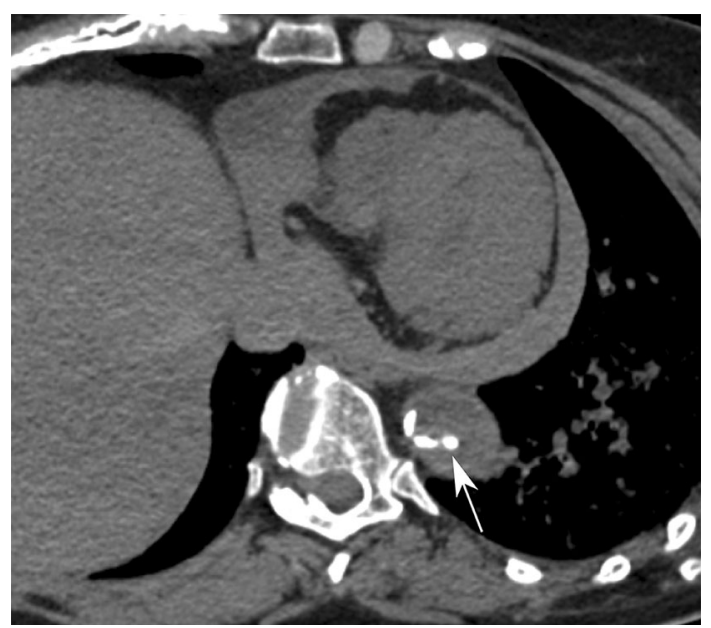

Fig. 1 Axial PMCT image of an aortic dissection case. The arrow indicates the dislocated calcified intima towards the aortic lumen. Note the concomitant hemopericardium

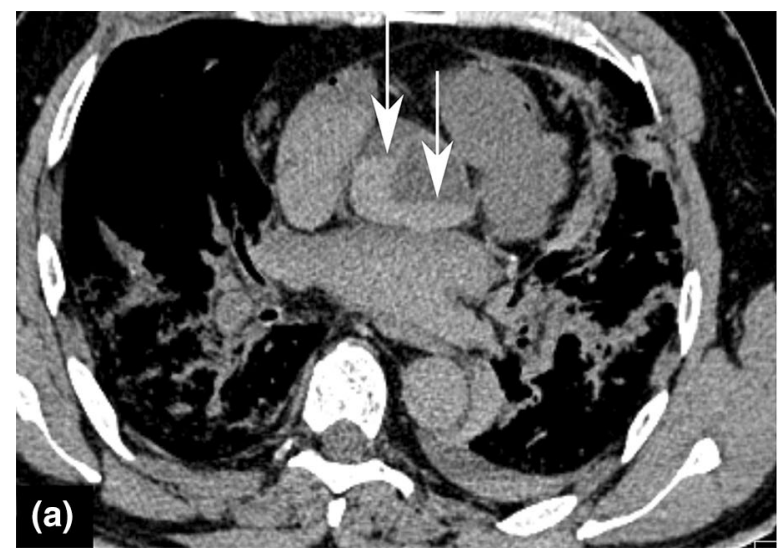

Fig. 2 Axial PMCT images of two distinct Stanford type A dissection cases. An intimomedial flap is recognizable in both cases, separating the true lumen from the false lumen. The double sedimentation sign can be identified in both cases as well. a The arrows indicate the different levels of sedimentation in the false and hemopericardium was noted (in two of these cases, a hemothorax was also present; one case exhibited a hemomediastinum), in 4/33 cases, a hemothorax was present (one also had a hemomediastinum and one had a retroperitoneal hemorrhage), and in another $1 / 33$, a retroperitoneal hemorrhage was observed. In 10/33 cases, no sign of hemorrhage or ruptured dissection could be recognized.

Aortic intimal calcification was present in 20/33 (61\%) cases.

Of the 20 cases with aortic calcification, the displaced calcification sign could be identified in $17(85 \%)$ cases by both readers in consensus [finding (2)]. The overall presence of the sign was $52 \%$ ( 17 out of 33 cases). The intrarater agreement was extremely high (Kappa 0.936, $p<0.0005)$, and the inter-rater agreement was substantial (Kappa 0.639, $p<0.0005$ ). The chi quadrat test confirmed the statistical significance $(p<0.0005)$.

An intimomedial flap [finding (3)] was present in 24/33 ( $73 \%$ ) cases. Of these 24 cases, 7 occurred in the absence of intimal calcification ( 7 out of 13 cases without calcification, $54 \%$ ). The remaining 17 are the same cases as those listed above, which also featured displaced intimal calcification. The intra-rater agreement was substantial (Kappa 0.727, $p<0.0005$ ), and the inter-rater agreement was fair (Kappa $0.333, p=0.01$ ).

Finding (4), the presence of double sedimentation (Fig. 2), was present in 16 out of the 33 (48\%) cases. This finding was only read in consensus. There was one case that lacked an identifiable intimomedial flap or dislocated calcification, which exhibited abnormal sedimentation.

The radiological consensus read-out of the described findings has been comprehensively illustrated in a flowchart (Fig. 3).

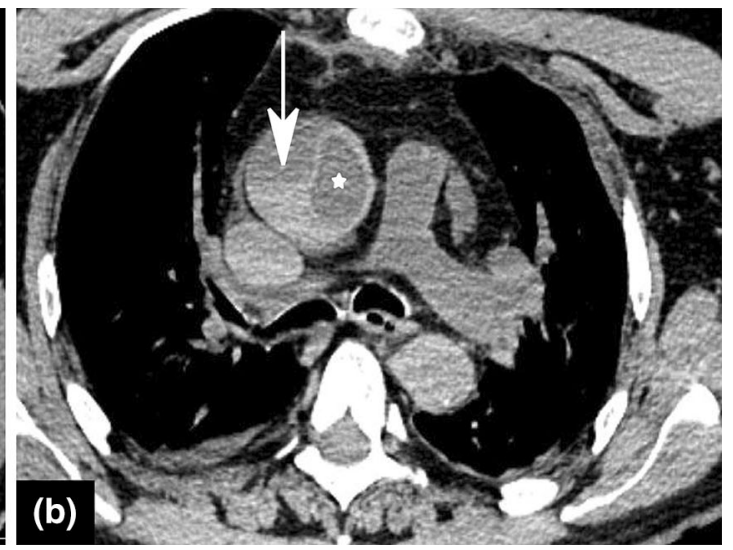

true lumen of the dissected aorta. Note the sedimented pleural effusion (hemothorax) of the left pleural cavity, the hemorrhage around the descending aorta, as well as the dislocated rib fracture on the left. b The true lumen (asterisk) presents no sedimentation, whereas the blood in the false lumen sediments (arrow) 


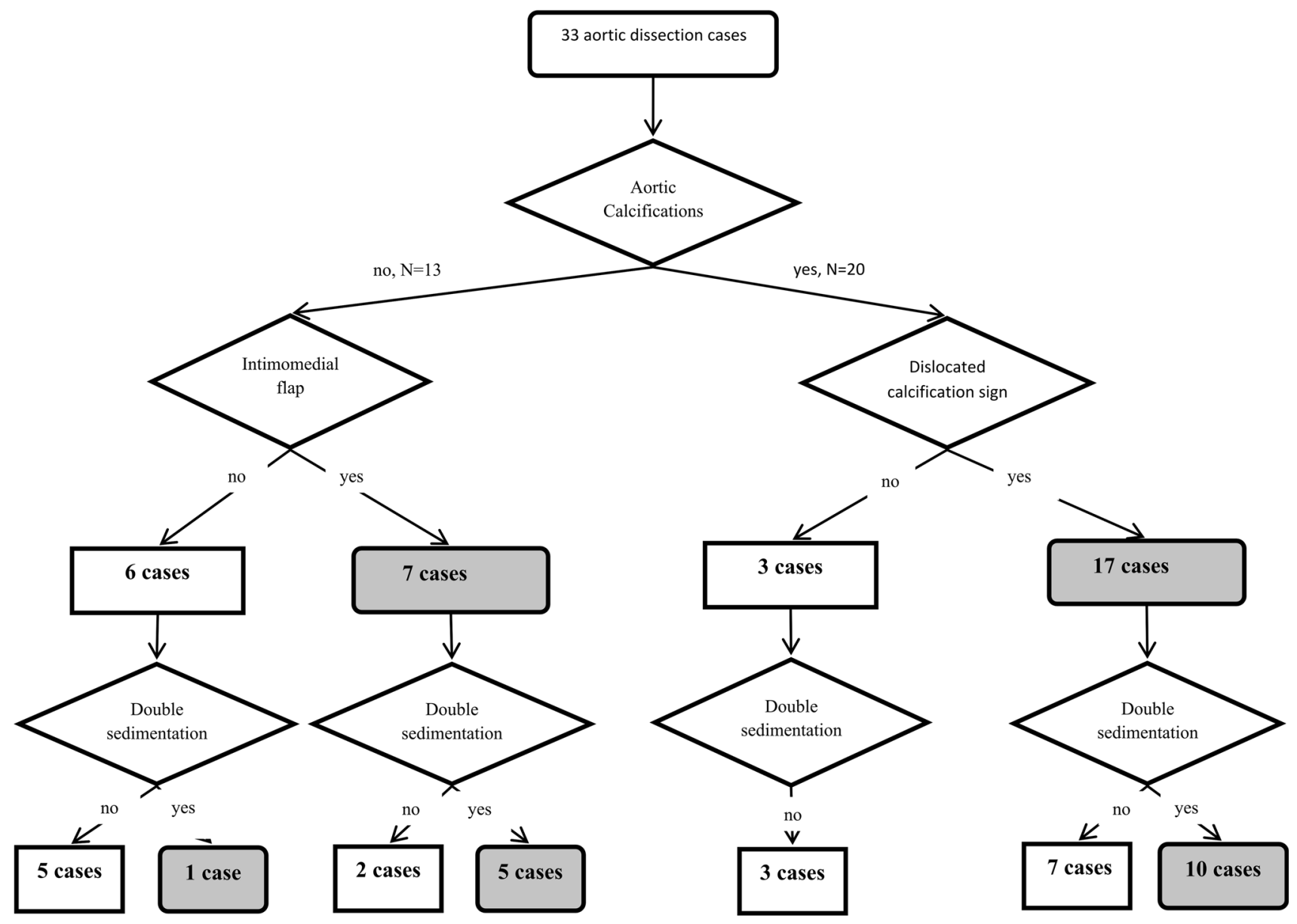

Fig. 3 Flowchart showing the prevalence of the described aortic dissection signs in PMCT images. A total of $85 \%$ (17/20) of the cases with aortic calcification exhibited dislocated calcification; whereas in approximately $54 \%(7 / 13)$ of the non-calcified aortas, the

\section{Discussion}

This study evaluated postmortem imaging findings using unenhanced PMCT examinations of aortic dissection cases. The radiologic signs of an aortic dissection were present in 25 out of the 33 cases (approximately $76 \%$ ) that were investigated.

Dislocated calcification is strongly indicative of aortic dissection. This finding concurs with the literature in several clinical cases $[6,10]$. However, dislocated calcification is a prerequisite to calcification of the aorta. It is interesting to note that among the 20 cases with aortic calcification, 17 exhibited this sign. Furthermore, this was shown to be statistically significant. In other words, if there was aortic calcification in a case with an aortic dissection, it was statistically more likely that you would also find displaced calcification.

The intimomedial flap was present in all of the cases with dislocated intima calcification, but it was also identified in cases without any aortic calcifications. The hyperattenuating intimomedial flap could be recognized. The double sedimentation sign was found in $48 \%(16 / 33)$ of the cases. Overall, in approximately $76 \%(25 / 33)$ of the cases, the described signs could be confirmed

aortic wall, which is a very common finding on PMCT due to the position-dependent sedimentation of the corpuscular blood cells (Shiotani et al. [23] described a $100 \%$ presence of this sign in their PMCT cases) may play a role in allowing visual identification. This is a possible explanation for why, in clinical radiology, an intimomedial flap is primarily described on contrast-enhanced CT images. According to Shiotani et al. [23], only $2 \%$ of the antemortem CT scans they examined had a hyperattenuating aortic wall. There are references of cases with a visible intimomedial flap in unenhanced antemortem, clinical CTs [31], however these patients were anemic [31].

The double sedimentation sign was found to co-exist with the abovementioned signs, and in the present study sample, there was one case where double sedimentation in the true and false lumen was identified in the absence of dislocated calcification or an intimomedial flap.

Postmortem sedimentation of the corpuscular blood particles is a well described phenomenon [24, 32]. The true 


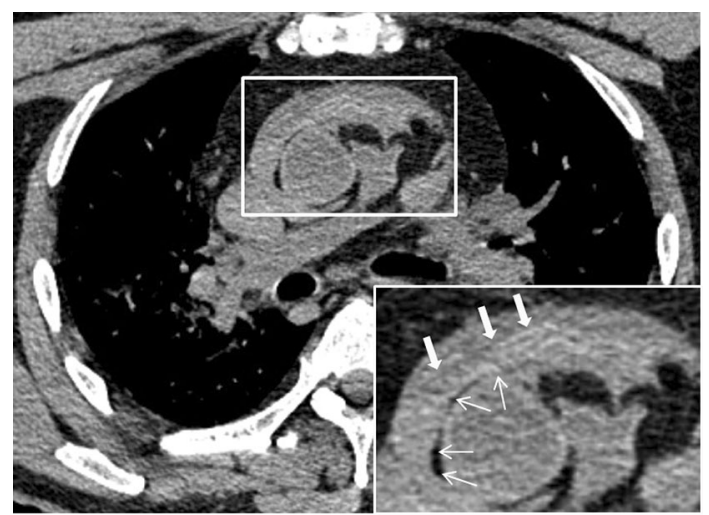

Fig. 4 A ruptured myocardial infarction with hemopericardium. The clotted blood in the pericardial sac is annotated (thick arrows). Neighboring the (non-dissected) ascending aorta, a thin layer of epicardial adipose tissue can be identified (thin arrows). This prevents confusing the hemopericardium with the double sedimentation sign and allows for exclusion of an aortic dissection, at least at that level

and false lumens of a dissected aorta form distinct spaces. The blood collects independently in each of them, leading to double (or more in cases of triple-lumen aorta) sedimentation.

The readers of the present study encountered a possible pitfall during the image interpretation of hemopericardium cases that must be mentioned. Investigators who use PMCT should be careful to not erroneously interpret the presence of extravasated blood in the pericardial sac as double sedimentation. Normally, a thin layer of epicardial fatty tissue exists between the aortic wall and the pericardial fluid, which allows for differentiation and exclusion of dissection, at least at that level (Fig. 4).

Another pitfall regarding the double sedimentation sign was noted in cases of normal sedimentation around a postmortem clot. A proper differentiation can be difficult to make; therefore, a double sedimentation sign should not be assumed to be present in the absence of a flap or dislocated calcification.

According to the literature, aortic dissections are found equally in the ascending and descending aorta [9], or they may be slightly in favor of type A dissections (60\%, [10]). However, in our study population, there were only 4 cases of type B dissection (4/33, $12 \%$ of the cases). A possible explanation for this is that type $\mathrm{A}$ is more often associated with fatal complications and is therefore considered a surgical emergency, whereas uncomplicated type B dissection is usually treated medically [4-7]. Partial thrombosis of the false lumen in type B aortic dissection cases is considered a strong independent predictor of mortality [33]. This inevitably explains why more Stanford type A dissection fatalities occur suddenly and are subject to forensic investigations, as they are considered to be unexpected deaths [34].
In approximately $24 \%(8 / 33)$ of the examined cases no signs of aortic dissection (dislocated calcification, intimomedial flap or double sedimentation) could be identified. This result is likely attributed to normal postmortem alterations in the vessel system. Collapse of the vessels is a normal postmortem finding [35] that is often observed in PMCT images, hampering the correct diagnosis of the aortic pathology. Decomposition also impedes the identification of aortic dissection signs, as the intra- and extravasal formation of gas prevents a definitive diagnosis.

The total number of cases included in the present study $(n=33)$ has to be mentioned as a further limitation. The power of the study would undoubtedly grow with a larger study population.

The absence of a control group in this study may also be considered as another limitation, however, it was purposely not included. The aim of the study was to describe the characteristic findings of aortic dissections in unenhanced PMCT images and to investigate how often these signs are encountered in such cases. It was not within the scope of this study to validate these findings as diagnostic criteria of aortic dissections.

\section{Conclusion}

The findings described in this study are commonly encountered in PMCT images of aortic dissection cases. In the 33 described cases, 25 exhibited one or more of the signs of this condition.

\section{Key Points}

1. $85 \%$ of the aortic dissection cases with aortic calcification presented the dislocated calcification sign; overall it was present in $52 \%$ of the cases.

2. An intimomedial flap could be recognized in $73 \%$ of the cases.

3. The double sedimentation sign, defined as either two distinct levels of sedimentation or as single sedimentation line within either the true or the false lumen, was present in $48 \%$ of the cases.

4. In total, aortic dissection findings on unenhanced PMCT could be confirmed in 25 out of 33 (76\%) autopsy confirmed aortic dissection cases.

\section{References}

1. Lovy AJ, Rosenblum JK, Levsky JM, Godelman A, Zalta B, Jain VR, Haramati LB. Acute aortic syndromes: a second look at dualphase CT. AJR. 2013;200:805-11. 
2. McMahon MA, Squirrell CA. FRCR Multidetector CT of aortic dissection: a pictorial review. Radiographics. 2010;30:445-60.

3. Lempel JK, Frazier AA, Jeudy J, Kligerman SJ, Schultz R, Ninalowo HA, Gozansky EK, Griffith B, White CS. Aortic arch dissection: a controversy of classification. Radiology. 2014;271(3): 848-55.

4. Loebe M, Ren D, Rodriguez L, La Francesca S, Bismuth J, Lumsden A. Acute and chronic thoracic aortic disease: surgical considerations. HSR Proc Intensive Care Cardiovasc Anesth. 2012;4(4):243-50.

5. Kapoor V, Ferris JV, Fuhrman CR. Intimomedial rupture: a new CT finding to distinguish true from false lumen in aortic dissection. AJR. 2004;183:109-12.

6. Yoo SM, Lee HY, White CS. MDCT evaluation of acute aortic syndrome. Radiol Clin N Am. 2010;48:67-83.

7. Bonaca MP, O'Gara PT. Diagnosis and management of acute aortic syndromes: dissection, intramural hematoma, and penetrating aortic ulcer. Curr Cardiol Rep. 2014;16(10):1-13.

8. Macura KJ, Corl FM, Fishman EK, et al. Pathogenesis in acute aortic syndromes: aortic dissection, intramural hematoma, and penetrating atherosclerotic aortic ulcer. Am J Roentgenol. 2003;181:309-16.

9. Coady MA, Rizzo JA, Elefteriades JA. Pathologic variants of thoracic aortic dissections penetrating atherosclerotic ulcers and intramural hematomas. Cardiol Clin. 1999;17(4):637-57.

10. Sebastià C, Pallisa E, Quiroga $S$, Alvarez-Castells A, Dominguez R, Evangelista A. Aortic dissection: diagnosis and follow up with helical CT. Radiographics. 1999;19:45-60.

11. Divchev D, Najjar T, Tillwich F, Aboukoura M, Rehders T, Nienaber CA. Risk assessment of acute aortic dissection. Dtsch Med Wochenschr. 2014;139(39):1947-51.

12. Meszaros I, Morocz J, Szlavi J, Schmidt J, Tornoci L, Nagy L, Szep L. Epidemiology and clinicopathology of aortic dissection. Chest. 2000;117:1271-8.

13. Proudfoot D, Shanahan CM. Biology of calcification in vascular cells: intima versus media. Herz. 2001;26:245-51.

14. Burke M, Parsons S, Bassed R. Management of medicolegal natural deaths from hemopericardium or hemothorax using postmortem CT scanning. Forensic Sci Med Pathol. 2012;8(4): 367-72.

15. Takahashi K, Stanford W. Multidetector CT of the thoracic aorta. Int J Cardiovasc Imaging. 2005;21:141-53.

16. Zeina AR, Trachtengerts V, Abadi S, Jarchowsky J, Soimu U, Nachtigal A. Thoraco-abdominal aorta dissection: look again before you leap. Radiol Case Rep. 2009;3(9):29-33.

17. Schyma C, Hagemeier L, Madea B. Bloodless aortic dissection. Forensic Sci Med Pathol. 2013;9:221-4

18. Sato Y, Satokawa H, Takase S, Misawa Y, Yokoyama H. Prolapse of aortic intimal flap into the left ventricle. A rare cause of global myocardial ischemia in acute type A aortic dissection. Circ J. 2006;70:214-5.

19. Baglivo M, Winklhofer S, Hatch GM, Ampanozi G, Thali MJ, Ruder TD. The rise of forensic and post-mortem radiologyAnalysis of the literature between the year 2000 and 2011. J Forensic Radiol Imaging. 2013;1:3-9.
20. Bedford PJ, Oesterhelweg L. Different conditions and strategies to utilize forensic radiology in the cities of Melbourne, Australia and Berlin, Germany. Forensic Sci Med Pathol. 2013;9:321-6.

21. Bolliger SA, Thali MJ, Ross S, Buck U, Naether S, Vock P. Virtual autopsy using imaging: bridging radiologic and forensic sciences. A review of the Virtopsy and similar projects. Eur Radiol. 2008;18(2):273-82.

22. Ross SG, Bolliger SA, Ampanozi G, Oesterhelweg L, Thali MJ, Flach PM. Postmortem CT angiography: capabilities and limitations in traumatic and natural causes of death. Radiographics. 2014;34:830-46.

23. Shiotani S, Kohno M, Ohashi N, Yamazaki K, Nakayama H, Ito Y, Kaga K, Ebashi T, Itai Y. Hyperattenuating aortic wall on postmortem computed tomography (PMCT). Radiat Med. 2002;20(4):201-6.

24. Shiotani S, Kohno M, Ohashi N, Yamazaki K, Itai Y. Postmortem intravascular high-density fluid level (hypostasis): CT findings. J Comput Assist Tomogr. 2002;26(6):892-3.

25. Thali MJ, Viner MD, Brogdon BG. Brogdon's forensic radiology. 2nd ed. Boca Raton: CRC Press; 2011.

26. Schwendener N, Mund M, Jackowski C. Type II DeBakey dissection with complete aortic rupture visualized by unenhanced postmortem imaging. Forensic Sci Int. 2013;225:67-70.

27. Lardi C, Lobrinus JA, Doenz F, Fracasso T, Augsburger M, Mangin P, Grabherr S. Acute aortic dissection with carotid and coronary malperfusion. From imaging to pathology. Am J Forensic Med Pathol. 2014;35:157-62.

28. Kluschke F, Ross S, Flach PM, Schweitzer W, Ampanozi G, Gascho D, Vonlanthen B, Thali MJ, Ruder TD. To see or not to see-Ambiguous findings on post-mortem cross-sectional imaging in a case of ruptured abdominal aortic aneurysm. Leg Med (Tokyo). 2013;15:256-9.

29. Flach PM, Gascho D, Schweitzer W, Ruder TD, Berger N, Ross SG, Thali MJ, Ampanozi G. Imaging in forensic radiology: an illustrated guide for postmortem computed tomography technique and protocols. Forensic Sci Med Pathol. 2014;10(4):583-606.

30. Hallinan JTPD, Anil G. Multi-detector computed tomography in the diagnosis and management of acute aortic syndromes. World J Radiol. 2014;6(6):355-65.

31. Demos TC, Posniak HV, Churchill RJ. Detection of the intimal flap of aortic dissection on unenhanced CT images. AJR. 1986;146:601-3.

32. Jackowski C, Thali M, Aghayev E, Yen K, Sonnenschein M, Zwygart K, Dirnhofer R, Vock P. Postmortem imaging of blood and its characteristics using MSCT and MRI. Int J Legal Med. 2006;120:233-40.

33. Tsai TT, Evangelista A, Nienaber CA, Myrmel T, Meinhardt G, Cooper JV, et al. Partial thrombosis of the false lumen in patients with acute type B aortic dissection. N Engl J Med. 2007;357(4):349-59.

34. Bailey K, Duflou J, Puranik R. Fatal cases of aortic dissection: an autopsy study. Int J Cardiol. 2012;158(1):148-9.

35. Christe A, Flach P, Ross S, Spendlove D, Bolliger S, Vock P, Thali MJ. Clinical radiology and postmortem imaging (Virtopsy) are not the same: specific and unspecific postmortem signs. Leg Med (Tokyo). 2010;12:215-22. 